\title{
Gauge-invariant theories and higher-degree forms
}

\section{S. Salgado}

Max-Planck-Institut für Physik, Föhringer Ring 6, 80805 Munich, Germany

Ludwig-Maximilians-Universität München, Theresienstraße 37, 80333 Munich, Germany

E-mail: salgado@mppmu.mpg.de

ABSTRACT: A free differential algebra is generalization of a Lie algebra in which the mathematical structure is extended by including of new Maurer-Cartan equations for higherdegree differential forms. In this article, we propose a generalization of the Chern-Weil theorem for free differential algebras containing only one $p$-form extension. This is achieved through a generalization of the covariant derivative, leading to an extension of the standard formula for Chern-Simons and transgression forms. We also study the possible existence of anomalies originated on this kind of structure. Some properties and particular cases are analyzed.

Keywords: Chern-Simons Theories, Differential and Algebraic Geometry, Gauge Symmetry

ARXIV EPRINT: 2108.02284 


\section{Contents}

1 Introduction 1

2 Free differential algebras $\quad 3$

3 Covariant derivative 5

4 Invariant density 1

4.1 Adjoint representation 8

5 Chern-Weil theorem 9

5.1 CS and transgression forms 9

$\begin{array}{ll}5.2 & \text { Extended Chern-Weil theorem }\end{array}$

6 Gauge anomalies 11

$\begin{array}{lll}6.1 & \text { Extended anomalies } & 12\end{array}$

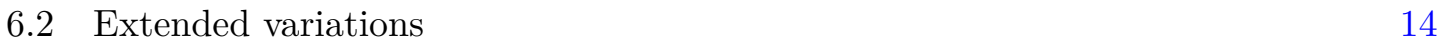

$\begin{array}{ll}\text { 6.3 Standard variations } & 16\end{array}$

$\begin{array}{lll}7 & \text { Concluding remarks } & 19\end{array}$

$\begin{array}{ll}\text { A Action for the Maxwell-FDA } & 19\end{array}$

B Subspace separation method 22

\section{Introduction}

Higher gauge theories are generalizations of the standard gauge theories that involve higherdegree differential forms. In the simplest case, this means introducing not only the usual one-form gauge connection but also a two-form gauge connection and a three-form fieldstrength, describing the parallel transport along surfaces. It is possible to continue such extension to gauge fields of degree higher than two, describing parallel transport along extended objects. In a higher gauge theory [1], the gauge potentials are locally represented as $p$-forms, whose corresponding $(p+1)$-form gauge curvatures allow the construction of action principles. The corresponding field equations are able to describe the dynamics of extended objects, such as $p$-branes, in a similar manner in which a standard gauge theory describes the dynamics of point particles. An example of this is found in $p$-form electrodynamics [2], whose gauge symmetry is described by the invariance under the transformation law $A \rightarrow A^{\prime}=A+\mathrm{d} \varphi$ where the abelian gauge field $A$ is a $p$-form, and $\varphi$ is a $(p-1)$ form and the parameter of the transformation. Another example of this type is the rank- 2 
abelian Kalb-Ramond gauge field [3]. A generalization to non-abelian higher-degree gauge theories has been studied on refs. [4-6]. In particular, in refs. [7-10], gauge-invariants forms were found, similar to the usual Chern-Pontryagin densities and their corresponding Chern-Simons (CS) forms for special cases.

From a physical point of view, it is interesting to note that a common feature to loop quantum gravity and string theory is the generalization of point particles to extended objects. It is then interesting to study the possible role that higher gauge theory could take in both frameworks.

In 1980 R. D'Auria, P. Fré and T. Regge [11] found an algebraic structure known as free differential algebra (FDA) or Cartan integrable system that allows formulating supergravity in the superspace in a geometric manner, representing the spacetime as a supermanifold. In 1982, R. D'Auria and P. Fré made use of such structure to unveil a hidden symmetry algebra in eleven-dimensional supergravity, previously constructed by Cremmer, Julia and Scherk [12, 13]. On the other hand, from ref. [14] it is known that the D'Auria-Fré formulation of supergravity is a higher-order geometric formulation of the Cartan supergeometry, where extended algebraic structures replace Poincaré Lie superalgebra.

First-order formulations of supergravity in six or more dimensions have a field content that includes bosonic higher-degree differential forms. Such field presence is a consequence of the consistence requirement of an equal number of bosonic and fermionic degrees of freedom in supersymmetry [15]. Since the field content of these theories cannot be encoded in one-forms dual to the generators of a Lie group, a possible solution is to replace the concept of group manifold used in the formulation of gravity and supergravity theories for a manifold that inherently involves higher degree forms. This led to introduce some mathematical structures in physics such as FDAs. These generalize the Maurer-Cartan equations that describe a Lie algebra but including higher-degree differential forms as potentials. FDAs are the natural generalization of Lie algebras, and since they include Lie algebras as subalgebras, they can be used to describe the field content of higher-dimensional gravity and supergravity theories.

The aim of this work is to use the FDA considered on refs. [16-19] to obtain a gaugeinvariant density and its corresponding CS form and study the presence of anomalies in this kind of theory. This paper is organized as follows: in section 2, we briefly review free differential algebras and their gauging, focused on the particular case which will be important in the results of this article. In section 3, we will propose a definition of covariant derivative that will be necessary for the construction of invariant gauge theories. In section 4 we introduce a generalization of the gauge invariant density of Lie algebras that includes the $p$-form of the already mentioned FDA and study the corresponding invariant tensor conditions. Section 5 contains a generalization of the Chern-Weil theorem with explicit expressions for transgression and CS actions for non-abelian gauge theory whose gauge fields are a one-form and a $p$-form $(p \geq 2)$. In section 6 we finish with a study on the existence of gauge anomalies for higher gauge theory, for which is also necessary to introduce new notation and study some mathematical properties about invariant tensors for FDAs. There are also two appendices with some useful properties and an application for gravity. 


\section{Free differential algebras}

The dual formulation of Lie algebras provided by the Maurer-Cartan equations can be naturally extended to $p$-forms. Let us consider a basis of differential forms $\left\{\Theta^{A(p)}\right\}_{p=1}^{N}$ defined on a manifold $M$ with $N \geq 2$. Each quantity $\Theta^{A(p)} \in \Lambda_{p}(M)$ is a differential $p$-form and the algebraic index $A(p)$ takes values on different sets depending on the value of $p$. Since $\left\{\Theta^{A(p)}\right\}_{p=1}^{N}$ is a basis, the exterior derivative $\mathrm{d} \Theta^{A(p)}$ can be written in terms of the same basis. This allows to write a set of Maurer-Cartan (MC) equations for a mathematical structure called free differential algebra

$$
\mathrm{d} \Theta^{A(p)}+\sum_{n=1}^{N} \frac{1}{n} C_{B_{1}\left(p_{1}\right) \cdots B_{n}\left(p_{n}\right)}^{A(p)} \Theta^{B_{1}\left(p_{1}\right)} \wedge \cdots \wedge \Theta^{B_{n}\left(p_{n}\right)}=0 .
$$

The coefficients $C_{B_{1}\left(p_{1}\right) \cdots B_{n}\left(p_{n}\right)}^{A(p)}$ are called generalized structure constants and are the generalization of the structure constants of Lie algebras to the case of FDAs. The nilpotent condition $\mathrm{d}^{2} \Theta^{A(p)}=0$ leads to the corresponding generalized Jacobi identity

$$
\begin{aligned}
\mathrm{d}^{2} \Theta^{A(p)} & =-\sum_{n, m=1}^{N} \frac{1}{m} C_{B_{1}\left(p_{1}\right) \cdots B_{n}\left(p_{n}\right)}^{A(p)} C_{D_{1}\left(q_{1}\right) \cdots D_{m}\left(q_{m}\right)}^{B_{1}\left(p_{1}\right)} \Theta^{D_{1}\left(q_{1}\right)} \wedge \cdots \wedge \Theta^{D_{m}\left(q_{m}\right)} \wedge \Theta^{B_{2}\left(p_{2}\right)} \wedge \cdots \wedge \Theta^{B_{n}\left(p_{n}\right)} \\
& =0 .
\end{aligned}
$$

We will restrict the analysis to a particular case that has been extensively studied in refs. $[16,17]$, in which the FDA is given by a Lie algebra with only one $p$-form extension, i.e., depending only on a 1 -form $\mu^{A}$ and a $p$-form $B^{i}$

$$
\begin{aligned}
\Theta^{A(1)} & =\mu^{A}, \\
\Theta^{A(p)} & =p ! B^{i}, \\
\Theta^{A(\text { others })} & =0,
\end{aligned}
$$

and therefore, reducing (2.1) to a set of two MC equations ${ }^{1}[16,17]$

$$
\begin{aligned}
\mathrm{d} \mu^{A}+\frac{1}{2} C_{B C}^{A} \mu^{B} \mu^{C} & =R^{A}=0, \\
\mathrm{~d} B^{i}+C_{A j}^{i} \mu^{A} B^{j}+\frac{1}{(p+1) !} C_{A_{1} \cdots A_{p+1}}^{i} \mu^{A_{1}} \cdots \mu^{A_{p+1}} & =R^{i}=0 .
\end{aligned}
$$

Eq. (2.7) shows how the FDA is an extension of the Lie algebra defined by eq. (2.6), achieved by the addition of a new MC equation for a $p$-form potential $B$ whose non-trivial structure is given by the presence of a cocycle

$$
\Omega^{i}=\frac{1}{(p+1) !} C_{A_{1} \cdots A_{p+1}}^{i} \mu^{A_{1}} \cdots \mu^{A_{p+1}}
$$

For consistency, this $(p+1)$-form must be covariantly closed. Otherwise, the second exterior derivative on eq. (2.7) does not vanish and the generalized Jacobi identity would not

\footnotetext{
${ }^{1}$ From now on we will omit the wedge product between differential forms.
} 
hold. Note that, if the cocycle is covariantly exact, i.e., $\Omega=\nabla \varphi$, it would be possible to write the Maurer-Cartan equation (2.7) as $\nabla B^{i}=0$, through a redefinition of the field $B^{i} \rightarrow B^{i}+\varphi$. Therefore, in order to have a non-trivial structure for the extended algebra, the cocycle must be covariantly closed but not covariantly exact. This means that, given a Lie algebra, there are as many non-equivalent FDA extensions as Chevalley-Eilenberg cohomology classes the Lie algebra has $[11,12]$.

To define gauge transformations using this algebra, we need to write the complete set of diffeomorphism transformations on the FDA manifold. Such diffeomorphisms are given by the Lie derivatives along all the possible directions on the FDA manifold. We need then to define a regular Lie derivative (as with Lie groups) and an extended one that determines the transformation of the $p$-form using a $(p-1)$-form parameter $\varepsilon^{j}$

$$
\begin{aligned}
& \ell_{\varepsilon^{A} t_{A}}=\mathrm{d} i_{\varepsilon^{A} t_{A}}+i_{\varepsilon^{A} t_{A}} \mathrm{~d},\text { (Lie derivative along the } \left.0 \text {-form } \varepsilon^{A} t_{A}\right), \\
&\left.\ell_{\varepsilon^{j} t_{j}}=\mathrm{d} i_{\varepsilon^{j} t_{j}}+i_{\varepsilon^{j} t_{j}} \mathrm{~d}, \quad \text { (Lie derivative along the }(p-1) \text {-form } \varepsilon^{j} t_{j}\right) .
\end{aligned}
$$

These derivatives are defined in terms of the contraction operators $i_{\varepsilon^{A} t_{A}}=\varepsilon^{A} i_{t_{A}}, i_{\varepsilon^{j} t_{j}}=$ $\varepsilon^{j} i_{t_{j}}$ whose action is defined in terms of the basis as follows

$$
\begin{aligned}
i_{t_{A}} \mu^{B} & =\delta_{A}^{B}, & i_{t_{A}} B^{j} & =0, \\
i_{t_{j}} \mu^{A} & =0, & i_{t_{j}} B^{i} & =\delta_{j}^{i} .
\end{aligned}
$$

Here, $t_{A}$ are the generators of the Lie algebra described by the eqs. (2.6) (subalgebra of the FDA). In the same way, $t_{j}$ correspond to a basis of vectors on the FDA manifold in the direction of the $p$-forms, i.e., $t_{j}$ is dual to $B^{j}$ in the same way in which $t_{A}$ is dual to $\mu^{A}[18,19]$. Applying the regular Lie derivative $\ell_{\varepsilon^{A} t_{A}}$ on the gauge fields $\mu^{A}$ and $B^{i}$ we have

$$
\begin{aligned}
& \ell_{\varepsilon^{A} t_{A}} \mu^{A}=\mathrm{d} \varepsilon^{A}+C_{B C}^{A} \mu^{B} \varepsilon^{C}+2 R_{B C}^{A} \varepsilon^{B} \mu^{C} \\
& \ell_{\varepsilon^{A} t_{A}} B^{i}=\left(R_{A j}^{i}-C_{A j}^{i}\right) \varepsilon^{A} B^{j}+\left((p+1) R^{i}{ }_{A A_{1} \cdots A_{p}}-\frac{1}{p !} C_{A A_{1} \cdots A_{p}}^{i}\right) \varepsilon^{A} \mu^{A_{1}} \cdots \mu^{A_{p}} .
\end{aligned}
$$

Applying the extended Lie derivative $\ell_{\varepsilon^{j} t_{j}}$ on the gauge fields $\mu^{A}$ and $B^{i}$ we have

$$
\begin{aligned}
& \ell_{\varepsilon^{j} t_{j}} \mu^{A}=\varepsilon^{j} R^{A}{ }_{j}, \\
& \ell_{\varepsilon^{j} t_{j}} B^{i}=\mathrm{d} \varepsilon^{j}+C_{A j}^{i} \mu^{A} \varepsilon^{j}-R^{i}{ }_{A j} \mu^{A} \varepsilon^{j} .
\end{aligned}
$$

Eqs. (2.12)-(2.15) contain the complete set of diffeomorphism transformations along all the independent directions of the FDA manifold $[18,19]$. Both transformation laws depend on the parameters $\varepsilon^{A}$ and $\varepsilon^{j}$ of the transformations and can be sumarized as follows

$$
\begin{aligned}
\delta \mu^{A}= & \mathrm{d} \varepsilon^{A}+C_{B C}^{A} \mu^{B} \varepsilon^{C}+2 R_{B C}^{A} \varepsilon^{B} \mu^{C}+\varepsilon^{j} R_{j}^{A}, \\
\delta B^{i}= & \mathrm{d} \varepsilon^{j}+C_{A j}^{i} \mu^{A} \varepsilon^{j}-R_{A j}^{i} \mu^{A} \varepsilon^{j}+\left(R_{A j}^{i}-C_{A j}^{i}\right) \varepsilon^{A} B^{j}+\left((p+1) R_{A A_{1} \cdots A_{p}}^{i}\right. \\
& \left.-\frac{1}{p !} C_{A A_{1} \cdots A_{p}}^{i}\right) \varepsilon^{A} \mu^{A_{1}} \cdots \mu^{A_{p}} .
\end{aligned}
$$


Note that these transformations also depend on the curvature of the FDA manifold. It is possible to obtain a restricted version of such transformations demanding horizontality of the curvatures in some directions of the FDA manifold, i.e.,

$$
\begin{aligned}
i_{\varepsilon^{B} t_{B}}\left(R_{C D}^{A} \mu^{C} \mu^{D}\right) & =0, \\
i_{\varepsilon^{i} t_{i}}\left(R_{j}^{A} B^{j}\right) & =0, \\
i_{\varepsilon^{B} t_{B}}\left(R_{A k}^{j} \mu^{A} B^{k}\right) & =0, \\
i_{\varepsilon^{i} t_{i}}\left(R_{A k}^{j} \mu^{A} B^{k}\right) & =0, \\
i_{\varepsilon^{B} t_{B}}\left(R_{A_{1} \cdots A_{p+1}}^{i} \mu^{A_{1}} \cdots \mu^{A_{p+1}}\right) & =0 .
\end{aligned}
$$

The curvature forms admit a splitting $R^{A}=R_{1}^{A}+R_{2}^{A}, R^{i}=R_{1}^{i}+R_{2}^{i}$ where

$$
\begin{aligned}
R_{1}^{A} & =R_{B C}^{A} \mu^{B} \mu^{C}, & R_{2}^{A} & =R_{j}^{A} B^{j}, \\
R_{1}^{i} & =R_{A_{1} \cdots A_{p+1}}^{i} \mu^{A_{1}} \cdots \mu^{A_{p+1}}, & R_{2}^{i} & =R_{A j}^{i} \mu^{A} B^{j},
\end{aligned}
$$

such that the horizontality conditions can be written as follows

$$
\begin{aligned}
i_{\varepsilon^{B} t_{B}} R_{1}^{A} & =i_{\varepsilon^{B} t_{B}} R_{1}^{i}=i_{\varepsilon^{B} t_{B}} R_{2}^{i}=0 \\
i_{\varepsilon^{j} t_{j}} R_{2}^{A} & =i_{\varepsilon^{j} t_{j}} R_{1}^{i}=0
\end{aligned}
$$

In a more convenient way, eqs. (2.24) and (2.25) can be written as

$$
i_{\varepsilon} R^{A}=0, \quad i_{\varepsilon^{B} t_{B}} R^{i}=0
$$

As happens with Lie groups, with these conditions, the diffeomorphisms become gauge transformations

$$
\begin{aligned}
& \delta \mu^{A}=\mathrm{d} \varepsilon^{A}+C_{B C}^{A} \mu^{B} \varepsilon^{C}, \\
& \delta B^{i}=\mathrm{d} \varepsilon^{i}+C_{A j}^{i} \mu^{A} \varepsilon^{j}-C_{A j}^{i} \varepsilon^{A} B^{j}-\frac{1}{p !} C_{A_{1} \cdots A_{p+1}}^{i} \varepsilon^{A_{1}} \mu^{A_{2}} \cdots \mu^{A_{p+1}} .
\end{aligned}
$$

Eq. (2.27) corresponds to the usual covariant derivative of the 0-form parameter. The second equation is the natural extension to the case of a $p$-form. Note that the transformation of $\mu^{A}$ is the same that appears in the study of standard gauge theory and it depends only on $\varepsilon^{A}$. On the other side, the transformation of $B^{i}$ depends on $\varepsilon^{A}$ and $\varepsilon^{i}[18,19]$.

\section{Covariant derivative}

In order to formulate a gauge theory involving $p$-forms whose invariance is governed by an FDA, it is necessary to define a covariant derivative that involves all the components of the connection. To find such derivative, it is useful to consider the information provided by the transformation law for the curvatures and the Bianchi identities. 
Using eqs. (2.27) and (2.28) it is possible to prove the following relations

$$
\begin{aligned}
\delta R^{A} & =C_{B C}^{A} R^{B} \varepsilon^{C}, \\
\delta R^{i} & =C_{A j}^{i} R^{A} \varepsilon^{j}-C_{A j}^{i} \varepsilon^{A} R^{j}-\frac{1}{(p-1) !} C_{A A_{1} \cdots A_{p}}^{i} \varepsilon^{A} R^{A_{1}} \mu^{A_{2}} \cdots \mu^{A_{p}} .
\end{aligned}
$$

Eq. (3.1) is equivalent to the Lie bracket between the 2-form curvature and the 0-form parameter. The natural generalization for the $(p+1)$-form curvature is expressed in eq. (3.2). We can see that $R^{i}$ also transforms homogeneously, i.e., not depending on derivatives of the parameters.

On the other hand, starting from the definition of the curvature forms, we can also calculate its exterior derivative to find the Bianchi identities

$$
\begin{aligned}
\mathrm{d} R^{A}-C_{B C}^{A} R^{B} \mu^{C} & =0, \\
\mathrm{~d} R^{i}+C_{A j}^{i} \mu^{A} R^{j}-C_{A j}^{i} R^{A} B^{j}-\frac{1}{p !} C_{A_{1} \cdots A_{p+1}}^{i} R^{A_{1}} \mu^{A_{2}} \cdots \mu^{A_{p+1}} & =0 .
\end{aligned}
$$

Since eq. (2.6) is the MC equation for a Lie algebra, it follows that eqs. (3.1) and (3.3) reproduce the variation of the curvature and Bianchi identity for Lie groups. Such relations and their corresponding extended versions are necessary to define the covariant derivative. However, we can see that the gauge transformations and Bianchi identities lead to different definitions of covariant derivatives.

From eqs, (2.27) and (2.28) we can see that if we have a set of differential forms $\varepsilon=\left(\varepsilon^{A}, \varepsilon^{i}\right)$, where $\varepsilon^{A}$ is a 0 -form and $\varepsilon^{i}$ is a $(p-1)$-form, then its covariant derivative is given by $\nabla \varepsilon=\left((\nabla \varepsilon)^{A},(\nabla \varepsilon)^{i}\right)$, with

$$
\begin{aligned}
& (\nabla \varepsilon)^{A}=\mathrm{d} \varepsilon^{A}+C_{B C}^{A} \mu^{B} \varepsilon^{C}, \\
& (\nabla \varepsilon)^{i}=\mathrm{d} \varepsilon^{i}+C_{A j}^{i} \mu^{A} \varepsilon^{j}-C_{A j}^{i} \varepsilon^{A} B^{j}-\frac{1}{p !} C_{A A_{1} \cdots A_{p}}^{i} \varepsilon^{A} \mu^{A_{1}} \cdots \mu^{A_{p}} .
\end{aligned}
$$

On the other hand, from eqs. (3.3) and (3.4) we can see that if we consider the curvature tensor of the FDA $R=\left(R^{A}, R^{i}\right)$ where $R^{A}$ is a 2 -form and $R^{i}$ is a $(p+1)$-form, then its covariant derivative is given by $\nabla R=\left((\nabla R)^{A},(\nabla R)^{i}\right)$, with

$$
\begin{aligned}
& (\nabla R)^{A}=\mathrm{d} R^{A}+C_{B C}^{A} \mu^{B} R^{C}, \\
& (\nabla R)^{i}=\mathrm{d} R^{i}+C_{A j}^{i} \mu^{A} R^{j}-C_{A j}^{i} R^{A} B^{j}-\frac{1}{p !} C_{A_{1} \cdots A_{p+1}}^{i} R^{A_{1}} \mu^{A_{2}} \cdots \mu^{A_{p+1}} .
\end{aligned}
$$

A possible answer is that these equations define the covariant derivative of every set of differential forms $x=\left(x^{A}, x^{i}\right)$ in the $(A, i)$-representation. However, there are two problems with this definition:

- It does not satisfy the homogeneity condition, i.e., the second covariant derivative $\nabla^{2} x$ depends on the exterior derivative of $x$.

- The gauge curvature does not satisfy $\delta R^{i}=\nabla \delta B^{i}$. 
At this point, we only know how to take the derivative of $\varepsilon$ and $R$. To solve this caveat, it is necessary to find a general definition that depends on the order of the differential forms of the corresponding set of fields. In the first case we have a set $\left(\varepsilon^{A}, \varepsilon^{i}\right)$ of $(0, p-1)$-forms and in the second one we have set $\left(R^{A}, R^{i}\right)$ of $(2, p+1)$-forms. In general, we have arrays of $(q, p+q-1)$-forms, and therefore, a general covariant derivative must be defined in terms of $q$. Let us introduce a $(q, p+q-1)$-set of differential forms in the $(A, i)$-representation of the algebra $x=\left(x^{A}, x^{i}\right)$. We propose that the covariant derivative of $x$ can be written in components as follows

$$
\begin{aligned}
& (\nabla x)^{A}=\mathrm{d} x^{A}+C_{B C}^{A} \mu^{B} x^{C}, \\
& (\nabla x)^{i}=\mathrm{d} x^{i}+C_{A j}^{i}\left(\mu^{A} x^{j}-(-1)^{f(q)} x^{A} B^{j}\right)-\frac{(-1)^{g(q)}}{p !} C_{A_{1} \cdots A_{p+1}}^{i} x^{A_{1}} \mu^{A_{2}} \cdots \mu^{A_{p+1}},
\end{aligned}
$$

where we have introduced the functions $f(q)$ and $g(q)$ in the terms involving the gauge fields and structure constants. From (3.6) and (3.8) we have

$$
(-1)^{f(0)}=(-1)^{f(2)}=(-1)^{g(0)}=(-1)^{g(2)}=1 .
$$

Besides, in order to satisfy the homogeneity condition, we need to remove the dependence on the derivatives of $x^{A}$ and $x^{i}$ in the second covariant derivatives of $x$. Those requirements lead to the following conditions

$$
(-1)^{f(q)}+(-1)^{f(q+1)}=0, \quad(-1)^{g(q)}+(-1)^{g(q+1)}=0,
$$

from which we have

$$
\begin{aligned}
& f(q+1)=f(q)+1, \\
& g(q+1)=g(q)+1 .
\end{aligned}
$$

A valid solution to this equation system is given by $f(q)=g(q)=q$, giving us the following definition

$$
\begin{aligned}
& (\nabla x)^{A}=\mathrm{d} x^{A}+C_{B C}^{A} \mu^{B} x^{C}, \\
& (\nabla x)^{i}=\mathrm{d} x^{i}+C_{A j}^{i}\left(\mu^{A} x^{j}-(-1)^{q} x^{A} B^{j}\right)-\frac{(-1)^{q}}{p !} C_{A_{1} \cdots A_{p+1}}^{i} x^{A_{1}} \mu^{A_{2}} \cdots \mu^{A_{p+1}} .
\end{aligned}
$$

Note that this definition satisfies the homogeneity condition, i.e., the second covariant derivative of $x$ does not depend on the derivatives on $x$. With this definition, we can write the variation of the gauge curvatures as

$$
\begin{aligned}
\delta R^{A} & =\nabla \delta \mu^{A}, \\
\delta R^{i} & =\nabla \delta B^{i} .
\end{aligned}
$$




\section{Invariant density}

Now we postulate an invariant density, analogous to the Chern-Pontryagin invariant of a Lie group. Using combinations of the curvavure forms, the most general $q$-form that can be written in terms of $R^{A}$ and $R^{i}$ is given by ${ }^{2}$

$$
\chi_{q}=\sum_{m, n \in q(p)} g_{A_{1} \cdots A_{m} i_{1} \cdots i_{n}} \underbrace{R^{A_{1}} \cdots R^{A_{m}}}_{2 m \text {-form }} \underbrace{R^{i_{1}} \cdots R^{i_{n}}}_{(p+1) n \text {-form }} .
$$

The constants $g_{A_{1} \cdots A_{m} i_{1} \cdots i_{n}}$ must be such that $\chi_{q}$ is gauge invariant, namely $\delta \chi_{q}=0$. The sum runs over all the possible values of $m$ and $n$ such that $2 m+(p+1) n=q$, and hence the resulting form is a $q$-form. Note the coefficients $g_{A_{1} \cdots A_{m} i_{1} \cdots i_{n}}$ contain mixed indices $A$ and $i$. Enforcing the gauge invariance condition $\delta \chi_{q}=0$ will constraint the form of such coefficients. Notice that the total variation of $\chi_{q}$ under gauge transformations is given by

$$
\begin{aligned}
\delta \chi_{q}= & \sum_{2 m+(p+1) n=q} g_{A_{1} \cdots A_{m} i_{1} \cdots i_{n}}\left[m C_{B C}^{A_{1}} R^{B} \varepsilon^{C} R^{A_{2}} \cdots R^{A_{m}} R^{i_{1}} \cdots R^{i_{n}}+n R^{A_{1}} \cdots R^{A_{m}}\left(C_{B j}^{i_{1}} R^{B} \varepsilon^{j}\right.\right. \\
& \left.\left.-C_{B j}^{i_{1}} \varepsilon^{B} R^{j}-\frac{1}{(p-1) !} C_{B B_{1} \cdots B_{p}}^{i_{1}} \varepsilon^{B} R^{B_{1}} \mu^{B_{2}} \cdots \mu^{B_{p}}\right) R^{i_{2}} \cdots R^{i_{n}}\right] .
\end{aligned}
$$

Since the parameters $\varepsilon^{A}, \varepsilon^{j}$ are independent, the terms proportional to each one have to vanish independently without imposing any extra condition on the fields and curvatures. This allows to split the independent conditions, resulting in the following three equations

$$
\begin{aligned}
& \sum_{r=1}^{m} C_{A_{0} A_{r}}^{C} g_{A_{1} \cdots \hat{A}_{r} C \cdots A_{m} i_{1} \cdots i_{n}}+\sum_{s=1}^{n} C_{A_{0} i_{s}}^{k} g_{A_{1} \cdots A_{m} i_{1} \cdots \hat{\imath}_{s} k \cdots i_{n}}=0, \\
& \sum_{r=1}^{m+1} C_{A_{r} B_{1} \cdots B_{p}}^{i_{1}} g_{A_{1} \cdots \hat{A}_{r} \cdots A_{m+1} i_{1} \cdots i_{n}}=0, \\
& \sum_{r=1}^{m+1} C_{A_{r} j}^{i_{1}} g_{A_{1} \cdots \hat{A}_{r} \cdots A_{m+1} i_{1} \cdots i_{n}}=0,
\end{aligned}
$$

where the indices with hat $\hat{A}$ and $\hat{\imath}$ denote the absence of $A$ and $i$ in the sequence. Eqs. (4.3)(4.5) correspond to the definition of extended invariant tensor for the FDA. Note that for $n=0$ (this is, in the absence of $p$-form extension), eq. (4.3) is equivalent to the standard definition of the invariant tensor of a Lie algebra. A short calculation shows that if the quantities $g_{A_{1} \cdots A_{m} i_{1} \cdots i_{n}}$ satisfy the invariant tensor conditions (4.3)-(4.5), then $\chi_{q}$ becomes a closed form, i.e., $\mathrm{d} \chi_{q}=0$.

\subsection{Adjoint representation}

An interesting case can be found when the $p$-form $B^{i}$ is also in the adjoint representation of the Lie subalgebra. In such case, the structure constants $C_{A k}^{i}$ become equivalent to the structure constants of the Lie subalgebra, i.e.,

$$
C_{A k}^{i} \rightarrow C_{A B}^{C}, \quad C_{A_{1} \cdots A_{p+1}}^{i} \rightarrow C_{A_{1} \cdots A_{p+1}}^{C} .
$$

\footnotetext{
${ }^{2}$ We define $q(p)$ as the set of non-negative integer solutions $(m, n)$ to the algebraic equation $2 m+$ $(p+1) n=q$.
} 
To avoid confusion with the indices of the invariant tensor, we introduce a comma to separate the indices corresponding to different sectors of the algebra $g_{A_{1} \cdots A_{m} i_{1} \cdots i_{n}} \rightarrow$ $g_{A_{1} \cdots A_{m}, B_{1} \cdots B_{n}}$. Note that the tensor is symmetric on the first set of indices but it still can be symmetric or antisymmetric on the second one depending on the value of $p$. In this case, the invariant tensor conditions (4.3)-(4.5) take the form

$$
\begin{array}{r}
\sum_{r=1}^{m} C_{A_{0} A_{r}}^{C} g_{A_{1} \cdots \hat{A}_{r} C \cdots A_{m}, B_{1} \cdots B_{n}}+\sum_{s=1}^{n} C_{A_{0} B_{s}}^{C} g_{A_{1} \cdots A_{m}, B_{1} \cdots \hat{B}_{s} C \cdots B_{n}} \\
=0, \\
\sum_{r=1}^{m+1} C_{A_{r} B_{1} \cdots B_{p}}^{C_{1}} g_{A_{1} \cdots \hat{A}_{r} \cdots A_{m+1} C_{1} \cdots C_{n}}=0, \\
\sum_{r=1}^{m+1} C_{A_{0} A_{r}}^{C_{1}} g_{A_{1} \cdots \hat{A}_{r} \cdots A_{m+1} C_{1} \cdots C_{n}}=0 .
\end{array}
$$

Eq. (4.7) is equivalent to the invariant tensor condition for Lie algebras. This makes it easier to find invariant tensors for FDAs; when the $p$-form is in the adjoint representation of the Lie subalgebra, an invariant tensor of the whole FDA is an invariant tensor of the Lie subalgebra that also satisfies the second and third conditions (4.8) and (4.9).

\section{Chern-Weil theorem}

Transgression and CS forms are chosen as Lagrangians for gravitational theories due to their invariance properties under gauge transformations. In particular, transgression forms are completely invariant and allow finding conserved charges. In the second order formalism of gravity, the fundamental field is the spacetime metric $g_{\mu \nu}$ and not the Levi-Civita connection $\Gamma_{\mu \nu}^{\lambda}$. The Levi-Civita connection is present in the formulation, but it is completely determined by the metric tensor. However, the first-order formalism allows one to consider both the metric and the connection as off-shell independent fields, each encoded in the components of one-forms evaluated in a Lie algebra. Since Chern-Simons and transgression theories are background-free theories that depend on a one-form gauge connection, they are good candidates to be considered gravitational theories, generalizing general relativity and introducing gauge invariance under a certain Lie group. See refs. [20, 21] for detailed reviews on the relation between invariant densities, CS and transgression forms, and physical theories. Gravitational theories in which the Lagrangians are CS forms and the invariance is described by space-time Lie groups, namely Poincaré and (Anti) de-Sitter groups, were proposed in refs. [22-24] for the three-dimensional case. In the last decade of the 20th century, A. Chamseddine extended CS forms to higher dimensions [25, 26]. The supersymmetrization of such models was introduced and extensively studied in refs. [27-32].

\subsection{CS and transgression forms}

The relation between CS and transgression forms emerges naturally in the Chern-Weil theorem: let $\mu$ and $\bar{\mu}$ be one-form gauge connections on a $2 n+1$ dimensional manifold, evaluated on a Lie algebra. Let $R=\mathrm{d} \mu+\mu^{2}$ and $\bar{R}=\mathrm{d} \bar{\mu}+\bar{\mu}^{2}$ their corresponding two-form curvatures. Then

$$
\operatorname{Tr} R^{n}-\operatorname{Tr} \bar{R}^{n}=\mathrm{d} Q_{2 n-1}(\mu, \bar{\mu}),
$$


where $\operatorname{Tr}$ denotes the trace over the elements of the Lie algebra on which the curvatures are valuated. The $(2 n-1)$-form $Q_{2 n-1}(\mu, \bar{\mu})$ is called transgression form and its explicit expression can be found by introducing a homotopic gauge field $\mu_{t}=\bar{\mu}+t(\mu-\bar{\mu})$ with its corresponding homotopic curvature $R_{t}=\mathrm{d} \mu_{t}+\mu_{t}^{2}$ depending on a parameter $t \in[0,1]$. Note that the homotopic parameter interpolates $\mu_{t}$ between $\bar{\mu}$ and $\mu$ and therefore, also $R_{t}$ between $\bar{R}$ and $R$. Then, it is possible to write down the transgression $(2 n-1)$-form as

$$
Q_{2 n-1}(\mu, \bar{\mu})=n \int_{0}^{1} \mathrm{~d} t \operatorname{Tr}\left[(\mu-\bar{\mu}) R_{t}^{n-1}\right] .
$$

By locally setting $\bar{\mu}=0$, the transgression form become a CS-form $Q_{2 n-1}(\mu)=Q_{2 n-1}(\mu, 0)$, satisfying the well-known relation

$$
\operatorname{Tr} R^{n}=\mathrm{d} Q_{2 n-1}(\mu) .
$$

Since the connection $\bar{\mu}$ cannot be globally fixed, the CS form can be only locally well defined.

\subsection{Extended Chern-Weil theorem}

In order to generalize the Chern-Weil theorem to the case of FDAs, let us consider the invariant density from eq. (4.1) for the homotopic gauge fields $\mu_{t}^{A}$ and $B_{t}^{i}$

$$
\begin{aligned}
\mu_{t}^{A} & =(1-t) \mu_{0}^{A}+t \mu_{1}^{A}, \\
B_{t}^{i} & =(1-t) B_{0}^{i}+t B_{1}^{i},
\end{aligned}
$$

with $t \in[0,1]$. Since the homotopic parameter $t$ interpolates between $\left(\mu_{0}, B_{0}\right)$ and $\left(\mu_{1}, B_{1}\right)$, the difference $\chi_{q}\left(\mu_{1}, B_{1}\right)-\chi_{q}\left(\mu_{0}, B_{0}\right)$ can be written as the following integral

$$
\chi_{q}\left(\mu_{1}, B_{1}\right)-\chi_{q}\left(\mu_{0}, B_{0}\right)=\int_{0}^{1} \mathrm{~d} t \frac{\mathrm{d}}{\mathrm{d} t} \chi_{q}\left(\mu_{t}, B_{t}\right) .
$$

Using the definition of covariant derivative for the FDA and performing the derivation inside of the integral, it is possible to show that the difference $\chi_{q}\left(\mu_{1}, B_{1}\right)-\chi_{q}\left(\mu_{0}, B_{0}\right)$ is an exact form

$$
\chi_{q}\left(\mu_{1}\right)-\chi_{q}\left(\mu_{0}\right)=\mathrm{d} Q_{q-1}\left(\mu_{1}, \mu_{0}\right),
$$

where the $(q-1)$-form $Q_{q-1}\left(\mu_{1}, \mu_{0}\right)$ is explicitly given by

$$
\begin{aligned}
Q_{q-1}\left(\mu_{1}, \mu_{0}\right)=\sum_{m, n \in q(p)} g_{A_{1} \cdots A_{m} i_{1} \cdots i_{n}} \int_{0}^{1} \mathrm{~d} t( & m u^{A_{1}} R_{t}^{A_{2}} \cdots R_{t}^{A_{m}} R_{t}^{i_{1}} \cdots R_{t}^{i_{n}} \\
& \left.+n R_{t}^{A_{1}} \cdots R_{t}^{A_{m}} u^{i_{1}} R_{t}^{i_{2}} \cdots R_{t}^{i_{n}}\right) .
\end{aligned}
$$

Here we define $u^{A}=\mu_{1}^{A}-\mu_{0}^{A}$ and $b^{i}=B_{1}^{i}-B_{0}^{i} . \quad R_{t}^{A}$ and $R_{t}^{i}$ are the curvatures corresponding to the homotopic gauge fields. For convenience, we rename $B^{i}=\mu^{i}, B_{t}^{i}=\mu_{t}^{i}$ and $b^{i}=u^{i}$. Then we can write $\mu=\left(\mu^{A}, \mu^{i}\right)$ as a single composite field and the same for the corresponding curvature. We can also write $\chi_{q}\left(\mu_{t}^{A}, B_{t}^{i}\right)=\chi_{q}\left(\mu_{t}^{A}, \mu_{t}^{i}\right)=\chi_{q}\left(\mu_{t}\right)$. This 
allows to write the generalization of the Chern-Weil theorem in a compact way that will be useful in future calculations

$$
\chi_{q}\left(\mu_{1}\right)-\chi_{q}\left(\mu_{0}\right)=\mathrm{d} Q_{q-1}\left(\mu_{1}, \mu_{0}\right) .
$$

As with the standard transgression forms, if we fix $\mu_{0}=\left(\mu_{0}^{A}, \mu_{0}^{i}\right)=(0,0)$ in eq. (5.8), we obtain the generalization of the Chern-Simons form to the case of FDAs. Analogously to the case with Lie algebras, this can be done only locally.

\section{Gauge anomalies}

The presence of anomalies in a theory is due to the breaking of classical symmetries in the quantization process. The chiral anomaly, introduced in refs. [33-36] appears in gauge theories that interact with Weyl fermions. The $U_{A}(1)$ or abelian anomaly is given by the divergence of the classically conserved current, and it is proportional to the ChernPontryagin 4-form

$$
\partial_{\mu} J^{\mu} \propto \operatorname{Tr}\left[\varepsilon_{\mu \nu \rho \sigma} R^{\mu \nu} R^{\rho \sigma}\right]=\operatorname{Tr}\left[\partial^{\mu} \varepsilon_{\mu \nu \rho \sigma}\left(\mu^{\nu} \partial^{\rho} \mu^{\sigma}+\frac{2}{3} \mu^{\nu} \mu^{\rho} \mu^{\sigma}\right)\right],
$$

where $\mu_{\nu}=\mu_{\nu}^{A} T_{A}$ is a gauge connection valuated on the Lie algebra of an internal Lie group with generators $t_{A}$ [37]. By introducing the one-form $\mu=\mu_{\mu}^{A} \mathrm{~d} x^{\mu} t_{A}$ and the exterior derivative $\mathrm{d}=\mathrm{d} x^{\mu} \partial_{\mu}$ it is possible to write the divergence (6.1) in terms of differential forms and the Hodge operator

$$
\mathrm{d} * J \propto \operatorname{Tr} R^{2}=\mathrm{d} Q_{3}(\mu)=\operatorname{Tr}\left[\mathrm{d}\left(\mu \mathrm{d} \mu+\frac{2}{3} \mu^{3}\right)\right],
$$

where $Q_{3}(\mu)$ is the standard CS 3-form.

On the other side, the so called non-abelian anomaly is given by the covariant divergence of a non-abelian current $J_{\mu A}$

$$
\mathrm{D}^{\mu} J_{\mu A} \propto \operatorname{Tr} \partial^{\mu}\left[t_{A} \varepsilon_{\mu \nu \rho \sigma}\left(\mu^{\nu} \partial^{\rho} \mu^{\sigma}+\frac{1}{2} \mu^{\nu} \mu^{\rho} \mu^{\sigma}\right)\right],
$$

or, in terms of differential forms

$$
\mathrm{D} * J_{A} \propto \mathrm{d}\left[\operatorname{Tr}\left(t_{A}\left(\mu \mathrm{d} \mu+\frac{1}{2} \mu^{3}\right)\right)\right] .
$$

In general, it is not possible to write the right-hand side of eqs. (6.4) in terms of gauge fields and field strengths. However, an interesting result due to B. Zumino in refs. [37, 38] shows that the non-abelian gauge anomaly can be derived from the gauge-variation of the CS form on eq. (6.2), which is porportional to the exterior derivative of certain two-form $Q_{2}^{1}(\varepsilon, \mu)$ depending on the gauge fields and the 0 -form parameter of the transformation $\varepsilon=\varepsilon^{A} T_{A}$

$$
\delta Q_{3}(\mu)=\mathrm{d} Q_{2}^{1}(\varepsilon, \mu)
$$


Those results are also valid in $2 n$-dimensional spacetimes, being in general possible to write down the abelian anomaly in terms of the total derivative of a $(2 n-1)$-dimensional CS form

$$
\mathrm{d} * J \propto \operatorname{Tr}\left(R^{n}\right)=\mathrm{d} Q_{2 n-1}(\mu) .
$$

In the same way, the non-abelian anomaly in a $(2 n-2)$-dimensional spacetime can derived from the $2 n$-dimensional abelian anomaly on eq. (6.6) by introducing a set of Lorentz-scalar fields $\varepsilon^{A}$ and computing the gauge variation of the $(2 n-1)$-dimensional CS form

$$
\delta Q_{2 n-1}(\mu)=\mathrm{d} Q_{2 n-2}^{1}(\varepsilon, \mu) .
$$

The $(2 n-2)$-form $Q_{2 n-2}^{1}(\varepsilon, \mu)$ has the following integral representation [37, 38]

$$
Q_{2 n-2}^{1}(\varepsilon, \mu)=n(n-1) \int_{0}^{1} \mathrm{~d} t(1-t) \operatorname{Str}\left(\varepsilon, \mathrm{d}\left(\mu, R_{t}^{n-2}\right)\right)
$$

where Str denotes the symmetrized trace over the algebraic elements.

\subsection{Extended anomalies}

Recently, it was found that it is possible to find gauge-invariant densities in the context of higher gauge theory. ${ }^{3}$ Moreover, in refs. $[7,8]$ it was studied the existence of gauge anomalies generated by such invariants. In this section we study the existence of anomalies, starting from the extended CS forms introduced in previous sections. Let us consider the following gauge field

$$
\mu=\left(\mu^{A}, \mu^{i}\right),
$$

consisting on one-forms $\mu^{A}$ and $p$-forms $\mu^{i}$. The corresponding curvature is given by a $(2, p+1)$-form $R=\left(R^{A}, R^{i}\right)$. As we have seen, a general field $X$ can be decomposed as a set $X=\left(X^{A}, X^{i}\right)$, where $X^{A}$ is a $r$-form and $X^{i}$ is a $\bar{r}$-form with $\bar{r}=r+p-1$. In such case we say that the array $X$ is of degree $r$. Let $X$ be an array of degree $x$ and $Y$ be an array of degree $y$. We introduce the following products between two arrays

$$
Z=\left(Z^{A}, Z^{i}\right)=[X, Y]
$$

where the components of $Z$ are given by

$$
\begin{aligned}
Z^{A} & =[X, Y]^{A}=C_{B C}^{A} X^{B} Y^{C}, \\
Z^{i} & =[X, Y]^{i}=C_{B j}^{i} X^{B} Y^{j} .
\end{aligned}
$$

Note that eqs. (6.11) and (6.12) imply that $Z^{A}$ is a $(x+y)$-form and $Z^{i}$ is a $(x+y+p-1)$ form.

Let us consider now the arrays $X_{r}$ and $Y_{s}$ of degree $x_{r}$ and $y_{s}$ respectively. We introduce the product between $p+1$ arrays with the following components

$$
\begin{aligned}
{\left[X_{1}, \ldots, X_{p+1}\right]^{A} } & =0, \\
{\left[X_{1}, \ldots, X_{p+1}\right]^{i} } & =C_{A_{1} \cdots A_{p+1}}^{i} X_{1}^{A_{1}} \cdots X_{p+1}^{A_{p+1}} .
\end{aligned}
$$

\footnotetext{
${ }^{3}$ See for instance refs. $[4-6,9,10]$.
} 
On the other hand, as we will see later, it is convenient to introduce a compact notation for the FDA invariant tensor. We denote

$$
\left\langle X_{1}, \ldots, X_{m} ; Y_{1}, \ldots, Y_{n}\right\rangle=g_{A_{1} \cdots A_{m} i_{1} \cdots i_{n}} X_{1}^{A_{1}} \cdots X_{m}^{A_{m}} Y_{1}^{i_{1}} \cdots Y_{n}^{i_{n}}
$$

This bracket separates the element before and after the semicolon, being the first ones evaluated in the Lie subalgebra and the latter on the extended sector. It is easy to check the following (anti)symmetry rules

$$
\begin{aligned}
& \left\langle\ldots, X_{r}, X_{r+1}, \ldots ; Y_{1}, \ldots, Y_{n}\right\rangle=(-1)^{x_{r} x_{r+1}}\left\langle\ldots, X_{r+1}, X_{r}, \ldots ; Y_{1}, \ldots, Y_{n}\right\rangle \\
& \left\langle X_{1}, \ldots, X_{m} ; \ldots, Y_{s}, Y_{s+1}, \ldots\right\rangle=(-1)^{\bar{y}_{s} \bar{y}_{s+1}+p+1}\left\langle X_{1}, \ldots, X_{m} ; \ldots, Y_{s+1}, Y_{s}, \ldots\right\rangle .
\end{aligned}
$$

Now we recall the invariant tensor conditions (4.3)-(4.5). Multiplying the first invariant tensor condition (4.3) by $\Theta^{A_{0}} X^{A_{1}} \cdots X^{A_{m}} Y^{i_{1}} \cdots Y^{i_{n}}$ where $\Theta^{A}$ is the first component of the degree- $\theta$ array $\Theta=\left(\Theta^{A}, \Theta^{i}\right)$ we get

$$
\begin{aligned}
& \sum_{r=1}^{m} \Theta^{A_{0}} X_{1}^{A_{1}} \cdots X_{m}^{A_{m}} Y_{1}^{i_{1}} \cdots Y_{n}^{i_{n}} C_{A_{0} A_{r}}^{C} g_{A_{1} \cdots \hat{A}_{r} C \cdots A_{m} i_{1} \cdots i_{n}} \\
& \quad+\sum_{s=1}^{n} \Theta^{A_{0}} X_{1}^{A_{1}} \cdots X_{m}^{A_{m}} Y_{1}^{i_{1}} \cdots Y_{n}^{i_{n}} C_{A_{0} i_{s}}^{k} g_{A_{1} \cdots A_{m} i_{1} \cdots \hat{\imath}_{s} k \cdots i_{n}}=0
\end{aligned}
$$

Using the new notation for the invariant tensor and the product given in eqs. (6.11) and (6.12) we obtain the following identity

$$
\begin{aligned}
& \sum_{r=1}^{m}(-1)^{\theta\left(x_{1}+\cdots+x_{r-1}\right)}\left\langle X_{1}, \ldots, X_{r-1},\left[\Theta, X_{r}\right], X_{r+1}, \ldots, X_{m} ; Y_{1}, \ldots, Y_{n}\right\rangle \\
& \quad+\sum_{s=1}^{n}(-1)^{\theta\left(x_{1}+\cdots+x_{m}+\bar{y}_{1}+\cdots \bar{y}_{s-1}\right)}\left\langle X_{1}, \ldots, X_{m} ; Y_{1}, \ldots, Y_{s-1},\left[\Theta, Y_{s}\right], Y_{s+1}, \ldots, Y_{n}\right\rangle=0 .
\end{aligned}
$$

In the same way, introducing a new set of arbitrary arrays $\Theta_{1}, \ldots, \Theta_{p}$ and multiplying (4.4) and (4.5) by $\Theta^{B_{1}} \cdots \Theta^{B_{p}} X^{A_{1}} \cdots X^{A_{m+1}} Y^{i_{2}} \cdots Y^{i_{n}}$ and $\Theta^{j} X^{A_{1}} \cdots X^{A_{m+1}} Y^{i_{2}} \cdots Y^{i_{n}}$ respectively we obtain the second and third invariant tensor conditions in terms of the new notation

$$
\begin{array}{r}
\sum_{r=1}^{m+1}(-1)^{x_{r}\left(x_{r+1}+\cdots+x_{m+1}\right)}\left\langle X_{1}, \ldots, X_{r-1}, X_{r+1}, \ldots, X_{m+1} ;\left[X_{r}, \Theta_{1}, \ldots, \Theta_{p}\right], Y_{2}, \ldots, Y_{n}\right\rangle=0 \\
\sum_{r=1}^{m+1}(-1)^{x_{r}\left(x_{r+1}+\cdots+x_{m+1}\right)}\left\langle X_{1}, \ldots, X_{r-1}, X_{r+1}, \ldots, X_{m+1} ;\left[X_{r}, \Theta\right], Y_{2}, \ldots, Y_{n}\right\rangle=0
\end{array}
$$

Eqs. (6.19)-(6.21) are the generalization of the invariant tensor property for Lie algebras, given in eq. (B.10) from ref. [37]. 
With the new notations, the Chern-Weil theorem for CS forms can be written in a more compact manner

$$
\chi_{q}(\mu)=\mathrm{d} Q_{q-1}(\mu)
$$

where

$$
Q_{q-1}(\mu)=\sum_{m, n \in q(p)} \int_{0}^{1} \mathrm{~d} t\left(m\left\langle\mu, R_{t}^{m-1} ; R_{t}^{n}\right\rangle+n\left\langle R_{t}^{m} ; \mu, R_{t}^{n-1}\right\rangle\right) .
$$

The purpose of this notation is to be able to find a gauge anomaly from the extended CS form in a more compact way. To achieve this, it is necessary to find an expression for the gauge variation of $Q^{(q-1)}(\mu)$ in terms of an exact form as with Lie groups

$$
\delta Q_{q-1}(\mu)=\mathrm{d} \omega_{q-2}^{1}(\varepsilon, \mu) .
$$

It is also convenient to separate the independent variations with respect to the parameters $\varepsilon^{A}$ and $\varepsilon^{i}$. Since the standard and extended transformations are independent, this leaves to two different generalizations of the gauge anomaly from eq. (6.8). Let us begin with the extended variation, i.e., the one proportional to $\varepsilon^{i}$.

\subsection{Extended variations}

The gauge variations of the gauge fields and curvatures parametrized by the $(p-1)$-form $\varepsilon^{i}$ are given by

$$
\begin{aligned}
\delta \mu^{A}=0, & \delta \mu^{i}=\mathrm{d} \varepsilon^{i}+[\mu, \varepsilon]^{i}, \\
\delta R^{A}=0, & \delta R^{i}=[R, \varepsilon]^{i} .
\end{aligned}
$$

Using the generalized Jacobi identity, it is direct to prove that the variation of the corresponding homotopic curvatures are

$$
\delta R_{t}^{A}=0, \quad \delta R_{t}^{i}=\left[R_{t}, \varepsilon\right]^{i}+t(t-1)[\mu, \mathrm{d} \varepsilon]^{i} .
$$

The variation of the CS form is then given by

$$
\begin{aligned}
\delta Q_{q-1}(\mu)= & \sum_{m, n} \int_{0}^{1} \mathrm{~d} t\left[n m\left\langle\mu, R_{t}^{m-1} ;\left[R_{t}, \varepsilon\right], R_{t}^{n-1}\right\rangle+n\left\langle R_{t}^{m} ;[\mu, \varepsilon], R_{t}^{n-1}\right\rangle+n\left\langle R_{t}^{m} ; \mathrm{d} \varepsilon, R_{t}^{n-1}\right\rangle\right. \\
& +t(t-1) m n\left\langle\mu, R_{t}^{m-1} ;[\mu, \mathrm{d} \varepsilon], R_{t}^{n-1}\right\rangle+n(n-1)\left\langle R_{t}^{m} ; \mu,\left[R_{t}, \varepsilon\right], R_{t}^{n-2}\right\rangle \\
& \left.+t(t-1) n(n-1)\left\langle R_{t}^{m} ; \mu,[\mu, \mathrm{d} \varepsilon], R_{t}^{n-2}\right\rangle\right] .
\end{aligned}
$$

From eqs. (6.19)-(6.21) it can be shown that the following relations hold

$$
\begin{array}{r}
\left\langle R_{t}^{m} ;\left[R_{t}, \varepsilon\right], \mu, R_{t}^{n-2}\right\rangle=0, \\
\left\langle R_{t}^{m} ;[\mu, \varepsilon], R_{t}^{n-1}\right\rangle+m\left\langle\mu, R_{t}^{m-1} ;\left[R_{t}, \varepsilon\right], R_{t}^{n-1}\right\rangle=0, \\
m\left\langle\left[\mu, R_{t}\right], R_{t}^{m-1} ; \mathrm{d} \varepsilon, \mu, R_{t}^{n-2}\right\rangle+\left\langle R_{t}^{m} ;[\mu, \mathrm{d} \varepsilon], \mu, R_{t}^{n-2}\right\rangle \\
+(-1)^{p}\left\langle R_{t}^{m} ; \mathrm{d} \varepsilon,[\mu, \mu], R_{t}^{n-2}\right\rangle+(n-2)\left\langle R_{t}^{m} ; \mathrm{d} \varepsilon, \mu,\left[\mu, R_{t}\right], R_{t}^{n-3}\right\rangle=0, \\
\left\langle[\mu, \mu], R_{t}^{m-1} ; \mathrm{d} \varepsilon, R_{t}^{n-1}\right\rangle-(m-1)\left\langle\mu,\left[\mu, R_{t}\right], R_{t}^{m-2} ; \mathrm{d} \varepsilon, R_{t}^{n-1}\right\rangle \\
-\left\langle\mu, R_{t}^{m-1} ;[\mu, \mathrm{d} \varepsilon], R_{t}^{n-1}\right\rangle+(-1)^{p+1}(n-1)\left\langle\mu, R_{t}^{m-1} ; \mathrm{d} \varepsilon,\left[\mu, R_{t}\right], R_{t}^{n-2}\right\rangle=0 .
\end{array}
$$


This allows to remove the terms including brackets between $\mu$ and $\mathrm{d} \varepsilon$ and write the gauge variation as follows

$$
\begin{aligned}
\delta Q_{q-1}(\mu)= & \sum_{m, n} \int_{0}^{1} \mathrm{~d} t t(t-1)\left[m n\left\langle[\mu, \mu], R_{t}^{m-1} ; \mathrm{d} \varepsilon, R_{t}^{n-1}\right\rangle\right. \\
& -m n(m-1)\left\langle\mu,\left[\mu, R_{t}\right], R_{t}^{m-2} ; \mathrm{d} \varepsilon, R_{t}^{n-1}\right\rangle \\
& +m n(n-1)(-1)^{p+1}\left\langle\mu, R_{t}^{m-1} ; \mathrm{d} \varepsilon,\left[\mu, R_{t}\right], R_{t}^{n-2}\right\rangle+n\left\langle R_{t}^{m} ; \mathrm{d} \varepsilon, R_{t}^{n-1}\right\rangle \\
& +n(n-1) m(-1)^{p}\left\langle\left[\mu, R_{t}\right], R_{t}^{m-1} ; \mathrm{d} \varepsilon, \mu, R_{t}^{n-2}\right\rangle+n(n-1)\left\langle R_{t}^{m} ; \mathrm{d} \varepsilon,[\mu, \mu], R_{t}^{n-2}\right\rangle \\
& \left.+n(n-1)(n-2)(-1)^{p}\left\langle R_{t}^{m} ; \mathrm{d} \varepsilon, \mu,\left[\mu, R_{t}\right], R_{t}^{n-3}\right\rangle\right] .
\end{aligned}
$$

The next step is to use the generalized Bianchi identities, the definition of the homotopic curvatures and eqs. (6.19)-(6.21) to obtain the following relations

$$
\begin{aligned}
{\left[\mu_{t}, R_{t}\right]^{A} } & =-\mathrm{d} R_{t}^{A}, \\
{\left[\mu_{t}, R_{t}\right]^{i} } & =-\mathrm{d} R_{t}^{i}+\left[R_{t}, \mu_{t}\right]^{i}+\frac{1}{p !}\left[R_{t}, \mu_{t}^{p}\right]^{i}, \\
t[\mu, \mu]^{A} & =\frac{\partial R_{t}^{A}}{\partial t}-\mathrm{d} \mu^{A}, \\
t[\mu, \mu]^{i} & =\frac{1}{2} \frac{\partial R_{t}^{i}}{\partial t}-\frac{1}{2} \mathrm{~d} \mu^{i}-\frac{t^{p}}{2 p !}\left[\mu^{p+1}\right]^{i}, \\
\left\langle R_{t}^{m} ;\left[\mu, \mu_{t}\right], \mathrm{d} \varepsilon, R_{t}^{n-2}\right\rangle & -m\left\langle\mu, R_{t}^{m-1} ; \mathrm{d} \varepsilon,\left[R_{t}, \mu_{t}\right], R_{t}^{n-2}\right\rangle=0, \\
\left\langle R_{t}^{m} ;\left[\mu, \mu_{t}\right], \mathrm{d} \varepsilon, R_{t}^{n-2}\right\rangle & +m\left\langle\mu, R_{t}^{m-1} ;\left[R_{t}, \mu_{t}\right], \mathrm{d} \varepsilon, R_{t}^{n-2}\right\rangle=0 .
\end{aligned}
$$

We use relations (6.34)-(6.39) to write the extended variation of $Q^{(q-1)}(\mu)$ in terms of the total derivatives $\partial / \partial t$ and $\mathrm{d}$

$$
\begin{aligned}
\delta Q_{q-1}(\mu) & =\sum_{m, n} \int_{0}^{1} \mathrm{~d} t\left[n \frac{\partial}{\partial t}\left\{(t-1)\left\langle R_{t}^{m} ; \mathrm{d} \varepsilon, R_{t}^{n-1}\right\rangle\right\}\right. \\
& -(t-1) m n(-1)^{(p+1)(n-1)}\left\langle\mathrm{d}\left(\mu, R_{t}^{m-1} ; R_{t}^{n-1}\right), \mathrm{d} \varepsilon\right\rangle \\
& \left.-(t-1)(-1)^{(p+1)(n-1)} n(n-1)\left\langle\mathrm{d}\left(R_{t}^{m} ; \mu, R_{t}^{n-2}\right), \mathrm{d} \varepsilon\right\rangle\right] .
\end{aligned}
$$

Note that the first term vanishes while the second and third terms are exact forms. This means that we can write the gauge variation of the Chern-Simons form (6.23) in terms of a $(q-2)$-form proportional to $\varepsilon^{i}$ which generalizes the expression for the non-abelian gauge anomaly

$$
\delta_{\text {Extended }} Q_{q-1}(\mu)=\mathrm{d} \omega_{q-2}^{1}\left(\varepsilon^{i}, \mu\right)
$$

where

$$
\omega_{q-2}^{1}\left(\varepsilon^{i}, \mu\right)=\sum_{m, n} \int_{0}^{1} \mathrm{~d} t(1-t) n\left(m\left\langle\mathrm{~d}\left(\mu, R_{t}^{m-1} ; R_{t}^{n-1}\right), \varepsilon\right\rangle+(n-1)\left\langle\mathrm{d}\left(R_{t}^{m} ; \mu, R_{t}^{n-2}\right), \varepsilon\right\rangle\right) .
$$




\subsection{Standard variations}

Let us consider now the standard variations of gauge fields and curvatures. This case presents some differences because, in order to obtain the anomaly term, we need to write the CS form in terms of new homotopic curvatures. Let us introduce some definitions before we proceed on this.

Given a degree-1 array $\mu=\left(\mu^{A}, \mu^{i}\right)$ of gauge fields, we have a corresponding degree-2 array of curvatures $R=\left(R^{A}, R^{i}\right)$. In terms of $\mu$ and $R$ we define the derivative operator [37]

$$
\begin{aligned}
\mathrm{d} \mu^{A} & =R^{A}-\frac{1}{2}[\mu, \mu]^{A}, \\
\mathrm{~d} \mu^{i} & =R^{i}-[\mu, \mu]^{i}-\frac{1}{(p+1) !}\left[\mu^{p+1}\right]^{i} .
\end{aligned}
$$

Using the Jacobi identities we can check that this definition verifies the nilpotent condition $\mathrm{d}^{2}=0$.

Now we consider an arbitrary variation on $\mu$ and $R$. With respect to that variation we introduce the homotopy operator $\ell$ such that its action on the arrays $\mu$ and $R$ is given by

$$
\ell \mu=0, \quad \ell R=\delta \mu .
$$

By direct inspection it can be shown that the homotopy operator $\ell$ satisfy the following anticommuting relations

$$
(\ell \mathrm{d}+\mathrm{d} \ell) \mu=\delta \mu, \quad(\ell \mathrm{d}+\mathrm{d} \ell) R=\delta R .
$$

Now we introduce new homotopic gauge fields. The one-form $\mu^{A}$ is parametrized as usual. However, the $p$-form $\mu^{i}$ is parametrized with a power of the parameter, such that $\mu_{t}$ still interpolates between $\mu_{0}$ and $\mu_{1}$ along a convenient trajectory in the parametric space

$$
\mu_{t}^{A}=t \mu^{A}, \quad \mu_{t}^{i}=t^{p} \mu^{i}
$$

If we consider a variation along the parameter $t$, then it is possible to define a homotopic operator $\ell_{t}$ with respect to such variation satisfying the relation $\ell \mathrm{d}+\mathrm{d} \ell=\mathrm{d}_{t}$

$$
\ell_{t} \mu_{t}=0, \quad \ell_{t} R_{t}=\mathrm{d}_{t} \mu_{t}=\mathrm{d} t \frac{\partial \mu_{t}}{\partial t} .
$$

Integrating the homotopic operator between 0 and 1 we find

$$
\int_{0}^{1}\left(\ell_{t} \mathrm{~d}+\mathrm{d} \ell_{t}\right)=\int_{0}^{1} \mathrm{~d}_{t}
$$

By applying the left-hand side of (6.49) into $\chi_{q}\left(\mu_{t}\right)$ we use Stokes' theorem and recover in this way the Chern-Weil theorem

$$
\int_{0}^{1}\left(\ell_{t} \mathrm{~d}+\mathrm{d} \ell_{t}\right) \chi_{q}\left(\mu_{t}\right)=\int_{0}^{1} \mathrm{~d} t \frac{\partial}{\partial t} \chi_{q}\left(\mu_{t}\right)=\chi_{q}(\mu)
$$

where

$$
\chi_{q}(\mu)=\mathrm{d} \int_{0}^{1} \ell_{t} \chi_{q}\left(\mu_{t}\right)=\mathrm{d} Q_{q-1}(\mu)
$$


A direct calculation of the action of the homotopy operator on $\chi_{q}\left(\mu_{t}\right)$ leads to an explicit expression of the CS form. Then we can write the generalized CS form in terms of the new homotopy (6.47)

$$
\begin{aligned}
Q_{q-1}(\mu) & =\int_{0}^{1} \ell_{t} \sum_{m, n}\left\langle R_{t}^{m} ; R_{t}^{n}\right\rangle \\
& =\sum_{m, n} \int_{0}^{1} \mathrm{~d} t\left(m\left\langle\mu, R_{t}^{m-1} ; R_{t}^{n}\right\rangle+n p t^{p-1}\left\langle R_{t}^{m} ; \mu, R_{t}^{n-1}\right\rangle\right) .
\end{aligned}
$$

This expression is equivalent to eq. (6.23), the difference being that it is written in terms of the new homotopy curvatures. The homotopic curvature $R_{t}^{A}$ remains the same while the extended one, as $\mu_{t}$, has a different dependency on $t$. Since the final expression is independent of the integration, it is natural to have different choices. The new expression must be chosen such that the anomaly is written in a more convenient way. In this case, the variation of the CS-form is given by

$$
\begin{aligned}
\delta Q_{q-1}(\mu)= & \sum_{m, n} \int_{0}^{1} \mathrm{~d} t\left[m\left\langle\delta \mu, R_{t}^{m-1} ; R_{t}^{n}\right\rangle+m(m-1)\left\langle\mu, \delta R_{t}, R_{t}^{m-2} ; R_{t}^{n}\right\rangle\right. \\
& +m n\left\langle\mu, R_{t}^{m-1} ; \delta R_{t}, R_{t}^{n-1}\right\rangle+m n p t^{p-1}\left\langle\delta R_{t}, R_{t}^{m-1} ; \mu, R_{t}^{n-1}\right\rangle \\
& \left.+n p t^{p-1}\left\langle R_{t}^{m} ; \delta \mu, R_{t}^{n-1}\right\rangle+n(n-1) p t^{p-1}\left\langle R_{t}^{m} ; \mu, \delta R_{t}, R_{t}^{n-2}\right\rangle\right] .
\end{aligned}
$$

The $\varepsilon^{A}$-gauge variation of the gauge fields and homotopic gauge curvatures is then given by

$$
\begin{aligned}
\delta \mu^{A} & =\mathrm{d} \varepsilon^{A}+[\mu, \varepsilon]^{A}, \\
\delta \mu^{i} & =-\left[\varepsilon, \mu_{t}\right]^{i}-\frac{1}{p !}\left[\varepsilon, \mu^{p}\right]^{i}, \\
\delta R_{t}^{A} & =\left[R_{t}, \varepsilon\right]^{A}+\left(t^{2}-t\right)[\mathrm{d} \varepsilon, \mu]^{A}, \\
\delta R_{t}^{i} & =-\left[\varepsilon, R_{t}\right]^{i}-\frac{t^{p-1}}{(p-1) !}\left[\varepsilon, R_{t}, \mu^{p-1}\right]^{i}+t^{p}(t-1)[\mathrm{d} \varepsilon, \mu]^{i}+\frac{t^{p}(t-1)}{p !}\left[\mathrm{d} \varepsilon, \mu^{p}\right]^{i} .
\end{aligned}
$$

A different choice of the homotopy leads to a more complicated expression for $\delta R_{t}^{i}$. This problem is not present in the previous case, and therefore, we only introduce the new homotopy rule for the anomaly resulting from the standard variations.

One more time we need to isolate the total derivative in eq. (6.53). To archieve this, we use eqs. (6.19)-(6.21) prove the following relations

$$
\begin{aligned}
& \left\langle[\mu, \mu], \mathrm{d} \varepsilon, R_{t}^{m-2} ; R_{t}^{n}\right\rangle-\left\langle\mu,[\mathrm{d} \varepsilon, \mu], R_{t}^{m-2} ; R_{t}^{n}\right\rangle \\
& \quad+(m-2)\left\langle\mu, \mathrm{d} \varepsilon,\left[\mu, R_{t}\right], R_{t}^{m-3} ; R_{t}^{n}\right\rangle+n\left\langle\mu, \mathrm{d} \varepsilon, R_{t}^{m-2} ;\left[\mu, R_{t}\right], R_{t}^{n-1}\right\rangle=0, \\
& \left\langle[\mathrm{~d} \varepsilon, \mu], R_{t}^{m-1} ; \mu, R_{t}^{n-1}\right\rangle-(m-1)\left\langle\mathrm{d} \varepsilon,\left[\mu, R_{t}\right], R_{t}^{m-2} ; \mu, R_{t}^{n-1}\right\rangle \\
& -\left\langle\mathrm{d} \varepsilon, R_{t}^{m-1} ;[\mu, \mu], R_{t}^{n-1}\right\rangle-(-1)^{p}(n-1)\left\langle\mathrm{d} \varepsilon, R_{t}^{m-1} ; \mu,\left[\mu, R_{t}\right], R_{t}^{n-2}\right\rangle=0, \\
& \left\langle\mu, R_{t}^{m-1} ;\left[\mathrm{d} \varepsilon, \mu^{p}\right], R_{t}^{n-1}\right\rangle-\left\langle\mathrm{d} \varepsilon, R_{t}^{m-1} ;\left[\mu^{p+1}\right], R_{t}^{n-1}\right\rangle \\
& -(m-1)\left\langle\mathrm{d} \varepsilon, \mu, R_{t}^{m-2} ;\left[R_{t}, \mu^{p}\right], R_{t}^{n-1}\right\rangle=0,
\end{aligned}
$$




$$
\begin{aligned}
&\left\langle\mu, R_{t}^{m-1} ;[\mathrm{d} \varepsilon, \mu], R_{t}^{n-1}\right\rangle-\left\langle\mathrm{d} \varepsilon, R_{t}^{m-1} ;[\mu, \mu], R_{t}^{n-1}\right\rangle \\
&-(m-1)\left\langle\mathrm{d} \varepsilon, \mu, R_{t}^{m-2} ;\left[R_{t}, \mu\right], R_{t}^{n-1}\right\rangle=0, \\
&\left\langle R_{t}^{m} ; \mu,[\mathrm{d} \varepsilon, \mu], R_{t}^{n-2}\right\rangle-(-1)^{p} m\left\langle\mathrm{~d} \varepsilon, R_{t}^{m-1} ;\left[R_{t}, \mu\right], \mu, R_{t}^{n-2}\right\rangle=0, \\
&\left\langle R_{t}^{m} ;\left[\mathrm{d} \varepsilon, \mu^{p}\right], \mu, R_{t}^{n-2}\right\rangle+m\left\langle\mathrm{~d} \varepsilon, R_{t}^{m-1} ;\left[R_{t}, \mu^{p}\right], \mu, R_{t}^{n-2}\right\rangle=0 .
\end{aligned}
$$

This allows one to eliminate the terms with a bracket of the type $[\mathrm{d} \varepsilon, \mu]$. The variation of the CS form takes then the form

$$
\begin{aligned}
& \delta Q_{q-1}(\mu) \\
& =\sum_{m, n} \int_{0}^{1} \mathrm{~d} t m\left[\left\langle\mathrm{~d} \varepsilon, R_{t}^{m-1} ; R_{t}^{n}\right\rangle+(t-1)\left((m-1) t(m-2)\left\langle\mu, \mathrm{d} \varepsilon,\left[\mu, R_{t}\right], R_{t}^{m-3} ; R_{t}^{n}\right\rangle\right.\right. \\
& +(m-1) \operatorname{tn}\left\langle\mu, \mathrm{d} \varepsilon, R_{t}^{m-2} ;\left[\mu, R_{t}\right], R_{t}^{n-1}\right\rangle+t^{p} n(m-1)\left\langle\mathrm{d} \varepsilon, \mu, R_{t}^{m-2} ;\left[R_{t}, \mu\right], R_{t}^{n-1}\right\rangle \\
& +\frac{t^{p}}{p !} n(m-1)\left\langle\mathrm{d} \varepsilon, \mu, R_{t}^{m-2} ;\left[R_{t}, \mu^{p}\right], R_{t}^{n-1}\right\rangle+n(n-1) p t^{2 p-1}(-1)^{p}\left\langle\mathrm{~d} \varepsilon, R_{t}^{m-1} ;\left[R_{t}, \mu\right], \mu, R_{t}^{n-2}\right\rangle \\
& +\frac{t^{p}}{p !} n(n-1) p t^{p-1}(-1)^{p}\left\langle\mathrm{~d} \varepsilon, R_{t}^{m-1} ;\left[R_{t}, \mu^{p}\right], \mu, R_{t}^{n-2}\right\rangle+n p t^{p-1} t(m-1)\left\langle\mathrm{d} \varepsilon,\left[\mu, R_{t}\right], R_{t}^{m-2} ; \mu, R_{t}^{n-1}\right\rangle \\
& +(m-1) t\left\langle[\mu, \mu], \mathrm{d} \varepsilon, R_{t}^{m-2} ; R_{t}^{n}\right\rangle+t^{p}(1+p) n\left\langle\mathrm{~d} \varepsilon, R_{t}^{m-1} ;\{[\mu, \mu]\}, R_{t}^{n-1}\right\rangle \\
& \left.\left.+\frac{t^{p}}{p !} n\left\langle\mathrm{~d} \varepsilon, R_{t}^{m-1} ;\left[\mu^{p+1}\right], R_{t}^{n-1}\right\rangle+(-1)^{p} n p t^{p-1} t(n-1)\left\langle\mathrm{d} \varepsilon, R_{t}^{m-1} ; \mu,\left[\mu, R_{t}\right], R_{t}^{n-2}\right\rangle\right)\right]
\end{aligned}
$$

Using one more time the definition of homotopic gauge curvatures and generalized Bianchi identities we get the following identities

$$
\begin{aligned}
t[\mu, \mu]^{A} & =\frac{\partial R_{t}^{A}}{\partial t}-\mathrm{d} \mu^{A}, \\
(p+1) t^{p}[\mu, \mu]^{i}+\frac{t^{p}}{p !}[\mu, \ldots, \mu]^{i} & =\frac{\partial R_{t}^{i}}{\partial t}-p t^{p-1} \mathrm{~d} \mu^{i}, \\
{\left[\mu_{t}, R_{t}\right]^{A} } & =-\mathrm{d} R_{t}^{A}, \\
{\left[\mu_{t}, R_{t}\right]^{i}-\left[R_{t}, \mu_{t}\right]^{i}-\frac{1}{p !}\left[R_{t}, \mu_{t}^{p}\right]^{i} } & =-\mathrm{d} R_{t}^{i} .
\end{aligned}
$$

Then, integrating by parts with respect to $\mathrm{d}$ and $\mathrm{d}_{t}$, we can explicitly write the variation of $Q_{q-1}(\mu)$ as an exact form, giving place to the anomaly proportional to the standard parameter $\varepsilon^{A}$

$$
\delta_{\text {Standard }} Q_{q-1}(\mu)=\mathrm{d} \omega_{q-2}^{1}\left(\varepsilon^{A}, \mu\right)
$$

where

$$
\omega_{q-2}^{1}\left(\varepsilon^{A}, \mu\right)=\sum_{m, n} \int_{0}^{1} \mathrm{~d} t(1-t) m\left\{(m-1)\left\langle\varepsilon, \mathrm{d}\left(\mu, R_{t}^{m-2} ; R_{t}^{n}\right)\right\rangle+n p t^{p-1}\left\langle\varepsilon, \mathrm{d}\left(R_{t}^{m-1} ; \mu, R_{t}^{n-1}\right)\right\rangle\right\} .
$$

Eqs. (6.42) and (6.70) determine the total gauge variation of the CS form in terms of both parameters $\varepsilon^{i}$ and $\varepsilon^{A}$ respectively. Note that the definition of $R_{t}$ is different in both cases due to the different choices of the homotopic gauge fields. 


\section{Concluding remarks}

In this article we have introduced a gauge invariant density for a particular FDA, analogous to the Chern-Pontryagin topological invariant for Lie algebras. Such FDA was introduced and studied on refs. [16-19] and its main feature is the presence of a Lie subalgebra and only one $p$-form extension through the inclussion of a non-trivial cocycle, representative of a Chevalley-Eilenberg cohomology class. Explicit expressions for transgression and CS forms whose integral representation is given by eq. (5.8) and possible gauge anomalies given by eqs. (6.42) and (6.70) were also found. The procedure to find those expressions is analogous to the one described in refs. $[37,38]$ in the calculation of non-trivial chiral anomalies. This is, by starting from the invariant density, obtain an integral expression for the corresponding CS form and finally, through its gauge variation, obtain the anomaly term. The main difference with respect to the standard case is the presence of extended indices. Since every expression contains both type of indices $A$ and $i$, being the first in the Lie subalgebra and the latter in the extended sector of the FDA, it is not possible to use the standard mathematical identities of group theory. In particular, the FDAs invariant tensor $g_{A_{1} \cdots A_{m} i_{1} \cdots i_{n}}$ does not satisfy the invariant tensor conditions of its Lie subalgebra unless $n=0$. To perform the calculations, it is therefore necessary to use its definition to obtain the generalized properties to which the invariant tensor obeys (6.19)-(6.21). Eqs. (6.42) and (6.70) are the generalization of the non-abelian anomaly (6.8), including non only one form gauge fields but also a $p$-form. Such extension of the field content in the theory is made in the same way in which the gauge symmetry is extended from a Lie algebra to an FDA through the inclusion of a non-trivial cocycle. Since the extension of the anomaly term has been found in a geometrical framework, its physical meaning must be separately studied in order to understand its possible consistency with the breaking of classical symmetries in QFT.

\section{Acknowledgments}

The autor would like to thank Laura Adrianopoli, Fabrizio Cordonier-Tello, Nicolás González, Dieter Lüst, Eduardo Rodríguez, Gustavo Rubio, Patricio Salgado and Mario Trigiante, for enlightening discussions. This research was partially funded by the bilateral DAAD-CONICYT grant 62160015. The author acknowledges the support from the Max-Planck-Society.

\section{A Action for the Maxwell-FDA}

Let us consider an extension of the bosonic Maxwell algebra. We introduce a set of gauge potentials $\mu=\left(\mu^{A}, B^{A}\right)$ whose components are a one-form $\mu^{A}=\left(e^{a}, \omega^{a b}, k^{a b}\right)$ and a threeform in the adjoint representation of Maxwell algebra $B^{A}=\left(b^{a}, b^{a b}, B^{a b}\right)$. By including a non-trivial cocycle $\Omega=\left(0,0, \Omega^{a b}\right)$ with

$$
\Omega^{a b}=k^{a}{ }_{c} k_{d}^{c} e^{d} e^{b}-k_{{ }_{c}}^{b} k^{c}{ }_{d} e^{d} e^{a}-2 k^{a b} k_{c d} e^{c} e^{d},
$$


it is possible to introduce the following Maurer-Cartan equations, defining an algebra that we call Maxwell-FDA

$$
\begin{aligned}
\mathrm{d} e^{a}+\omega^{a}{ }_{c} e^{c} & =0, \\
\mathrm{~d} \omega^{a b}+\omega^{a}{ }_{c} \omega^{c b} & =0, \\
\mathrm{~d} k^{a b}+\omega^{a}{ }_{c} k^{c b}-\omega^{b}{ }_{c} k^{c a}+\frac{1}{l^{2}} e^{a} e^{b} & =0, \\
\mathrm{~d} b^{a}+\omega^{a}{ }_{c} b^{c}+b^{a}{ }_{c} e^{c} & =0, \\
\mathrm{~d} b^{a b}+\omega^{a}{ }_{c} b^{b c}-\omega^{b}{ }_{c} b^{b a} & =0, \\
\mathrm{~d} B^{a b}+\omega^{a}{ }_{c} B^{c b}-\omega^{b}{ }_{c} B^{c a}+b^{a}{ }_{c} k^{c b}-b^{b}{ }_{c} k^{c a}+\frac{1}{l^{2}}\left(e^{a} b^{b}-e^{b} b^{a}\right)+\Omega^{a b} & =0 .
\end{aligned}
$$

By gauging the algebra we consider non-zero curvatures. For convenience we denote the 2-form curvature components as $R^{A}=\left(R^{a}, R^{a b}, F^{a b}\right)$ and, since $B^{i}$ is in the adjoint representation, we denote the 4 -form curvature as $R^{i} \rightarrow H^{A}=\left(h^{a}, h^{a b}, H^{a b}\right)$. This allows us to propose the following gauge invariant 6 -form

$$
\chi_{6}(\mu, B)=g_{A B C} R^{A} R^{B} R^{C}+g_{A B} R^{A} H^{B} .
$$

In this case, the invariant tensor conditions (4.7)-(4.9) are given by

$$
\begin{aligned}
g_{A D} C_{B C}^{D} R^{A} R^{B} \varepsilon^{C} & =0, \\
g_{A D} C_{B C}^{A} R^{B} \varepsilon^{C} H^{D}-g_{A D} C_{B C}^{D} R^{A} \varepsilon^{B} H^{C} & =0, \\
g_{A B} C_{C D E F}^{B} R^{A} \varepsilon^{C} R^{D} \mu^{E} \mu^{F} & =0 .
\end{aligned}
$$

As we have seen, in this case, the condition (A.9) means that $g_{A B}$ is also an invariant tensor of the Lie subalgebra. The second condition becomes equivalent to the first one, while the third condition is given by

$$
g_{A D} C_{B C}^{D}+g_{B D} C_{A C}^{D}=0 .
$$

Since $g_{A B}$ is an invariant tensor of the Maxwell algebra, we propose the usual rank-2 invariant tensor ${ }^{4}$

$$
\begin{aligned}
g_{a b, c d} & =\alpha_{0}\left(\eta_{a c} \eta_{b d}-\eta_{a d} \eta_{b c}\right), \\
g_{a b,[c d]} & =\alpha_{1}\left(\eta_{a c} \eta_{b d}-\eta_{a d} \eta_{b c}\right), \\
g_{a b, c} & =\alpha_{2} \epsilon_{a b c}, \\
g_{a, b} & =\alpha_{1} \eta_{a b},
\end{aligned}
$$

where $\alpha_{0}, \alpha_{1}$ and $\alpha_{2}$ are arbitrary constants. However, this tensor still has to verify eq. (A.11) in order to be an invariant tensor of the whole FDA. By imposing that condition, we find $\alpha_{1}=\alpha_{2}=0$. The rank-2 invariant tensor of Maxwell-FDA is then given by (A.13).

\footnotetext{
${ }^{4}$ We denote with square brackets the indices in the $k^{a b}$ sector of the algebra. In this case, it doesn't mean antisymmetrization.
} 
From (5.8) we can write down a Chern-Simons action given by

$$
Q_{5}(\mu)=3 \int_{0}^{1} \mathrm{~d} t g_{A B C} \mu^{A} R_{t}^{B} R_{t}^{C}+g_{A B} \int_{0}^{1} \mathrm{~d} t\left(B^{A_{1}} H_{t}^{B}+R_{t}^{A} B^{B}\right) .
$$

However, in order to obtain a simpler expression, it is convenient to introduce a new set of gauge fields and use the triangle relation given by eq. (B.9) in appendix B. We define $\bar{\mu}=\left(\bar{\mu}^{A}, \bar{B}^{A}\right)$ whose components are given as follows

$$
\begin{aligned}
& \bar{\mu}^{A}=\left(0, \omega^{a b}, k^{a b}\right), \\
& \bar{B}^{A}=\left(0, b^{a b}, B^{a b}\right) .
\end{aligned}
$$

Then we can write the CS form (A.17) in terms of a transgression form, another CS form and total derivatives

$$
Q_{5}(\mu)=Q_{5}(\mu, \bar{\mu})+Q_{5}(\bar{\mu})+\text { total derivative. }
$$

From eq. (5.8) we know that the explicit expression for $Q_{5}(\mu, \bar{\mu})$ is

$$
Q_{5}(\mu, \bar{\mu})=\int_{0}^{1} \mathrm{~d} t\left(3 g_{A B C}\left(\mu^{A}-\bar{\mu}^{A}\right) R_{t}^{B} R_{t}^{C}+g_{A B}\left[\left(\mu^{A}-\bar{\mu}^{A}\right) H_{t}^{B}+R_{t}^{A_{1}}\left(B^{B}-\bar{B}^{B}\right)\right]\right) .
$$

In this case, the homotopic gauge fields are given by $\mu_{t}=\bar{\mu}+t(\mu-\bar{\mu})$ where indexwise $\mu_{t}$ is given by

$$
\mu_{t}^{A}=\left(t e^{a}, \omega^{a b}, k^{a b}\right), \quad \mu_{t}^{i}=\left(t b^{a}, b^{a b}, B^{a b}\right) .
$$

On the other hand, the CS form $Q_{5}(\bar{\mu})$ is given by

$$
Q_{5}(\bar{\mu})=\int_{0}^{1} \mathrm{~d} t g_{A B}\left(\bar{\mu}^{A} \bar{H}_{t}^{B}+\bar{R}_{t}^{A} \bar{B}^{i}\right)
$$

with the homotopic gauge field $\bar{\mu}_{t}=t \bar{\mu}=\left(t \bar{\mu}^{A}, t \bar{B}^{B}\right)$ and $\bar{R}_{t}=\left(\bar{R}_{t}^{A}, \bar{H}_{t}^{B}\right)$ being its corresponding curvature. Using the rank-3 invariant tensor of the Maxwell algebra $g_{a b, c d, e}=$ $\epsilon_{a b c d e}$ and the rank-2 invariant tensor (A.13) for the extended sector, we obtain the following CS form

$$
Q_{5}(\mu)=\frac{3}{4} \epsilon_{a b c d e} R^{a b} R^{c d} e^{e}+\frac{\alpha_{0}}{2}\left(\omega^{a b} h_{a b}+R^{a b} b_{a b}\right) .
$$

The first term corresponds to the usual CS form invariant under the transformations of Maxwell Lie algebra. The second term extends the Lagrangian, including the threeform gauge field without breaking the invariance under transformations of the FDA and modifying the resulting dynamics in the corresponding theory. However, it is important to note that the cocycle is not present in the Lagrangian. Since the only non-vanishing component of the invariant tensor in the extended sector of the algebra is given by (A.13), the $B^{a b}$ field is not present in the CS form. To find an example of a CS Lagrangian invariant under the transformations of an FDA and involving a non-trivial cocycle in the transformations is still an open problem. 


\section{B Subspace separation method}

Extended CS and transgression forms satisfy similar invariance conditions that their standard versions. There is also a triangle relation between them that can be explicitly found using the extended Cartan homotopy formula (ECHF) [20, 21, 39].

Let us consider a set of $r+2$ composite gauge connections $\left\{\mu_{J}=\left(\mu_{J}^{A}, \mu_{J}^{i}\right)\right\}_{J=0}^{r+1}$ defined on a fiber bundle over $M$ and a $(r+1)$ dimensional simplex $T_{r+1}$ with $r+2$ parameters $t_{J} \in[0,1]$, satisfying the constraint $\sum_{J} t_{J}=1$. It is possible to defined an homotopic connection $\mu_{t}=\sum_{J} t_{J} \mu_{J}$ whose components transform according (2.27), (2.28). The ECHF is given by

$$
\int_{\partial T_{r+1}} \frac{\ell_{t}^{s}}{s !} \chi=\int_{T_{r+1}} \frac{\ell_{t}^{s+1}}{(s+1) !} \mathrm{d} \chi+(-1)^{s+q^{\prime}} \mathrm{d} \int_{T_{r+1}} \frac{\ell_{t}^{s+1}}{(s+1) !} \chi
$$

where, in this case, $\chi$ represents any polynomial in the forms $\left\{\mu_{t}^{A}, \mu_{t}^{i}, R_{t}^{A}, R_{t}^{i}, \mathrm{~d}_{t} \mu_{t}^{A}, \mathrm{~d}_{t} \mu_{t}^{i}\right.$, $\left.\mathrm{d}_{t} R_{t}^{A}, \mathrm{~d}_{t} R_{t}^{i}\right\}$ which is a $q$-form on $M$ and a $q^{\prime}$-form on $T_{r+1}$, with $q \geq s$ and $s+q^{\prime}=r$. The exterior derivatives on $M$ and $T_{r+1}$ are denoted respectively by $\mathrm{d}$ and $\mathrm{d}_{t}$ and the homotopy operator $\ell_{t}$, which now is defined with respect to the variations along the simplex, maps mixed differential forms according to

$$
\begin{gathered}
\ell_{t}: \Lambda^{a}(M) \times \Lambda^{b}\left(T_{r+1}\right) \rightarrow \Lambda^{a-1}(M) \times \Lambda^{b+1}\left(T_{r+1}\right), \\
\ell_{t} \mu_{t}=0, \quad \ell_{t} R_{t}=\mathrm{d}_{t} \mu_{t}
\end{gathered}
$$

i.e., $\ell_{t}$ increases the order of the differential form on $T_{r+1}$ and decreases the order on $M$ while it satisfies Leibniz's rule as well as $\mathrm{d}$ and $\mathrm{d}_{t}$. Note that the ECHF is different for any value of $s$. However, its allowed values are $s=0, \ldots q$. As happens in the case studied in section 6 , the operators $\mathrm{d}, \mathrm{d}_{t}$ and $\ell_{t}$ define a graded algebra given by [39]

$$
\begin{aligned}
\mathrm{d}^{2} & =0, \quad \mathrm{~d}_{t}^{2}=0, \quad\left\{\mathrm{~d}, \mathrm{~d}_{t}\right\}=0, \\
{\left[\ell_{t}, \mathrm{~d}\right] } & =\mathrm{d}_{t}, \quad\left[\ell_{t}, \mathrm{~d}_{t}\right]=0 .
\end{aligned}
$$

In this case, we consider the closed polynomial $\chi=\chi_{q}\left(\mu_{t}\right)$, reducing the ECHF to

$$
\int_{\partial T_{s+1}} \frac{\ell_{t}^{s}}{s !} \chi_{q}=(-1)^{s} \mathrm{~d} \int_{T_{s+1}} \frac{\ell_{t}^{s+1}}{(s+1) !} \chi_{q}
$$

By setting $s=0$, the homotopic connection is given by $\mu_{t}=\left(\mu_{t}^{A}, \mu_{t}^{i}\right)=\mu_{0}+t\left(\mu_{1}-\mu_{0}\right)$ and then eq. (B.6) reproduces the generalized Chern-Weil theorem

$$
\begin{aligned}
\chi_{q}\left(\mu_{1}\right)-\chi_{q}\left(\mu_{0}\right)=\mathrm{d} \sum_{m, n \in q(p)} g_{A_{1} \cdots A_{m} i_{1} \cdots i_{n}} \int_{T_{1}}\{ & m\left(\ell_{t} R_{t}^{A_{1}}\right) R_{t}^{A_{1}} \cdots R_{t}^{A_{m}} R_{t}^{i_{1}} \cdots R_{t}^{i_{n}} \\
& \left.+n R_{t}^{A_{1}} \cdots R_{t}^{A_{m}}\left(\ell_{t} R_{t}^{i_{1}}\right) R_{t}^{i_{2}} \cdots R_{t}^{i_{n}}\right\} .
\end{aligned}
$$

For $s=1$ we have a three-dimensional simplex, the homotopic gauge field is given by $\mu_{t}=t^{0}\left(\mu_{0}-\mu_{1}\right)+t^{2}\left(\mu_{2}-\mu_{1}\right)+\mu_{1}$ and eq. (B.6) takes the form

$$
\int_{\partial T_{2}} \ell_{t} \chi_{q}\left(\mu_{t}\right)=-\mathrm{d} \int_{T_{2}} \frac{\ell_{t}^{2}}{2} \chi_{q}\left(\mu_{t}\right) .
$$


Performing the integration directly over $T_{2}$ and $\partial T_{2}$ we find a triangle relation for the generalized transgression forms

$$
Q_{q-1}\left(\mu_{1}, \mu_{2}\right)-Q_{q-1}\left(\mu_{0}, \mu_{2}\right)+Q_{q-1}\left(\mu_{0}, \mu_{1}\right)=\mathrm{d} Q_{q-2}\left(\mu_{2}, \mu_{1}, \mu_{0}\right) .
$$

Note that if we choose $\mu_{0}=\left(\mu_{0}^{A}, \mu_{0}^{i}\right)=(0,0)$ we obtain an expression that relates the transgression form with two CS forms and a total derivative. This equation is useful in explicit calculations of CS and transgression actions.

Open Access. This article is distributed under the terms of the Creative Commons Attribution License (CC-BY 4.0), which permits any use, distribution and reproduction in any medium, provided the original author(s) and source are credited.

\section{References}

[1] J.C. Baez and J. Huerta, An Invitation to Higher Gauge Theory, Gen. Rel. Grav. 43 (2011) 2335 [arXiv: 1003.4485] [INSPIRE].

[2] C. Teitelboim, Gauge Invariance for Extended Objects, Phys. Lett. B 167 (1986) 63 [INSPIRE].

[3] B. Zwiebach, A first course in String Theory, Cambridge University Press (2004).

[4] G. Savvidy, Non-abelian tensor gauge fields. I, Int. J. Mod. Phys. A 21 (2006) 4931 [INSPIRE].

[5] G. Savvidy, Non-Abelian tensor gauge fields: Generalization of Yang-Mills theory, Phys. Lett. $B 625$ (2005) 341 [hep-th/0509049] [INSPIRE].

[6] S. Konitopoulos and G. Savvidy, Propagating modes of non-Abelian tensor gauge field of second rank, J. Phys. A 41 (2008) 355402 [arXiv:0706.0762] [InSPIRE].

[7] I. Antoniadis and G. Savvidy, New gauge anomalies and topological invariants in various dimensions, Eur. Phys. J. C 72 (2012) 2140 [arXiv:1205.0027] [InSPIRE].

[8] I. Antoniadis and G. Savvidy, Extension of Chern-Simons forms and new gauge anomalies, Int. J. Mod. Phys. A 29 (2014) 1450027 [arXiv:1304.4398] [InSPIRE].

[9] S. Konitopoulos and G. Savvidy, Extension of Chern-Simons forms, J. Math. Phys. 55 (2014) 062304 [arXiv:1401.4812] [INSPIRE].

[10] P. Salgado and S. Salgado, Extended gauge theory and gauged free differential algebras, Nucl. Phys. B 926 (2018) 179 [arXiv:1702.07819] [inSPIRE].

[11] R. D'Auria, P. Fré and T. Regge, Graded Lie Algebra Cohomology and Supergravity, Riv. Nuovo Cim. 3N12 (1980) 1 [INSPIRE].

[12] R. D'Auria and P. Fré, Geometric Supergravity in d=11 and Its Hidden Supergroup, Nucl. Phys. B 201 (1982) 101 [Erratum ibid. 206 (1982) 496] [INSPIRE].

[13] E. Cremmer, B. Julia and J. Scherk, Supergravity Theory in Eleven-Dimensions, Phys. Lett. B 76 (1978) 409 [INSPIRE].

[14] P.K. Townsend, M theory from its superalgebra, NATO Sci. Ser. C 520 (1999) 141 [hep-th/9712004] [INSPIRE]. 
[15] L. Castellani, R. D'Auria and P. Fre, Supergravity and superstrings: A Geometric perspective. Vol. 2: Supergravity, World Scientific, Singapore (1991), pp. 607-1371.

[16] L. Castellani and A. Perotto, Free differential algebras: Their use in field theory and dual formulation, Lett. Math. Phys. 38 (1996) 321 [hep-th/9509031] [INSPIRE].

[17] L. Castellani, Lie Derivatives along Antisymmetric Tensors, and the M-theorySuperalgebra, J. Phys. Math. 3 (2011) P110504 [hep-th/0508213] [InSPIRE].

[18] L. Castellani, Extended Lie derivatives and a new formulation of $D=11$ supergravity, J. Phys. Math. 3 (2011) P110505 [hep-th/0604213] [InSPIRE].

[19] L. Castellani, Higher form gauge fields and their nonassociative symmetry algebras, JHEP 09 (2014) 055 [arXiv:1310.7185] [INSPIRE].

[20] F. Izaurieta, E. Rodriguez and P. Salgado, Eleven-dimensional gauge theory for the $M$ algebra as an Abelian semigroup expansion of osp(32|1), Eur. Phys. J. C 54 (2008) 675 [hep-th/0606225] [INSPIRE].

[21] P. Mora, R. Olea, R. Troncoso and J. Zanelli, Transgression forms and extensions of Chern-Simons gauge theories, JHEP 02 (2006) 067 [hep-th/0601081] [INSPIRE].

[22] P. van Nieuwenhuizen, D = 3 Conformal Supergravity and Chern-Simons Terms, Phys. Rev. D 32 (1985) 872 [INSPIRE].

[23] A. Achucarro and P.K. Townsend, A Chern-Simons Action for Three-Dimensional anti-de Sitter Supergravity Theories, Phys. Lett. B 180 (1986) 89 [InSPIRE].

[24] E. Witten, (2+1)-Dimensional Gravity as an Exactly Soluble System, Nucl. Phys. B 311 (1988) 46 [INSPIRE].

[25] A.H. Chamseddine, Topological Gauge Theory of Gravity in Five-dimensions and All Odd Dimensions, Phys. Lett. B 233 (1989) 291 [InSPIRE].

[26] A.H. Chamseddine, Topological gravity and supergravity in various dimensions, Nucl. Phys. B 346 (1990) 213 [INSPIRE].

[27] M. Bañados, R. Troncoso and J. Zanelli, Higher dimensional Chern-Simons supergravity, Phys. Rev. D 54 (1996) 2605 [gr-qc/9601003] [INSPIRE].

[28] J. Zanelli, Quantization of the gravitational constant in odd dimensional gravity, Phys. Rev. D 51 (1995) 490 [hep-th/9406202] [INSPIRE].

[29] R. Troncoso and J. Zanelli, New gauge supergravity in seven-dimensions and eleven-dimensions, Phys. Rev. D 58 (1998) 101703 [hep-th/9710180] [INSPIRE].

[30] R. Troncoso and J. Zanelli, Gauge supergravities for all odd dimensions, Int. J. Theor. Phys. 38 (1999) 1181 [hep-th/9807029] [INSPIRE].

[31] R. Troncoso and J. Zanelli, Higher dimensional gravity, propagating torsion and AdS gauge invariance, Class. Quant. Grav. 17 (2000) 4451 [hep-th/9907109] [INSPIRE].

[32] M. Hassaine, R. Troncoso and J. Zanelli, 11D supergravity as a gauge theory for the M-algebra, PoS WC2004 (2005) 006 [hep-th/0503220] [INSPIRE].

[33] J.S. Schwinger, On gauge invariance and vacuum polarization, Phys. Rev. 82 (1951) 664 [INSPIRE].

[34] L. Rosenberg, Electromagnetic interactions of neutrinos, Phys. Rev. 129 (1963) 2786 [INSPIRE]. 
[35] S.L. Adler and D.G. Boulware, Anomalous commutators and the triangle diagram, Phys. Rev. 184 (1969) 1740 [INSPIRE].

[36] S.L. Adler and W.A. Bardeen, Absence of higher order corrections in the anomalous axial vector divergence equation, Phys. Rev. 182 (1969) 1517 [INSPIRE].

[37] B. Zumino, Y.-S. Wu and A. Zee, Chiral Anomalies, Higher Dimensions, and Differential Geometry, Nucl. Phys. B 239 (1984) 477 [InSPIRE].

[38] B. Zumino, Chiral Anomalies and Differential Geometry, in Les Houches Summer School on Theoretical Physics: Relativity, Groups and Topology, pp. 1291-1322 (1983).

[39] J. Mañes, R. Stora and B. Zumino, Algebraic Study of Chiral Anomalies, Commun. Math. Phys. 102 (1985) 157 [INSPIRE]. 